\title{
The First Comprehensive Study of H-Deficient Phenotypes in Iran
}

\author{
Ehsan Shahverdi ${ }^{a}$ b $\quad$ Mostafa Moghaddam ${ }^{\mathrm{a}}$ Bashir Hajbeigi ${ }^{\mathrm{a}} \quad$ Ali Akbar Pourfathollah $^{\mathrm{a}, \mathrm{c}}$ \\ Fatemeh Hassani $^{\mathrm{a}}$ Fahimeh Herfat ${ }^{\mathrm{a}}$ \\ a Blood Transfusion Research Center, High Institute for Research and Education in Transfusion Medicine, Tehran, Iran; \\ ${ }^{b}$ Blood and Cancer Research Center, MAHAK Pediatric Cancer Treatment and Research Center, Tehran, Iran; \\ 'Faculty of Medical Sciences, Tarbiat Modares University, Tehran, Iran
}

Keywords

Anti-H antibody $\cdot \mathrm{H}$-deficient (Bombay) $\cdot \mathrm{H}$ antigen $\cdot$ Iran

\section{Summary}

Background: The lack of correct blood grouping practices can lead to missing of the rare Bombay Oh phenotype and subjecting patients to the risk of severe hemolytic transfusion reaction. In the absence of blood donor registry, transfusion management of patients is a challenge. We performed this study in order to estimate the prevalence of the Bombay blood group (Oh) in Iran and to determine whether consanguinity plays a role in the prevalence of Oh group. Methods: This is a descriptive study in the Immunohematology Reference Laboratory of the Iranian Blood Transfusion Organization (IBTO) Tehran, Iran, over a period of 7 years. All donor blood samples showing blood group $\mathrm{O}$ and a strong initial reaction with blood group O RBC control cells were tested with anti-H lectin. Also blood samples from blood group $\mathrm{O}$ patients were tested with anti-H lectin if all cells on both antibody screening tests and antibody identification panels were reactive with negative auto control test. Specialized tests like adsorption/elution technique and inhibition assay for determination of secretor status were performed on Oh cases. Any history of consanguineous marriages were recorded. All variables were categorical variables, and percentage and proportions were calculated manually. Results: Analysis of the results of over 7 million first-time blood donors in Iran showed that the most common ABO blood group was O, with 2,520,000 (36\%) subjects. 56 Oh individuals' (donors and patients) phenotypes (0.0008\%) were detected. Consanguinity was observed in 50 cases (89\%). Conclusions: This study shows that the prevalence of Bombay blood group in the general population of Iran is relatively high $(0.0008 \%)$ and associated with consanguineous marriage. Thus, consanguinity is still an important risk factor present.

(c) 2018 S. Karger GmbH, Freiburg

\section{Introduction}

The serum of individuals with Oh (Bombay) blood type contain strong anti-H in addition to anti-A and anti-B [1]. If patients with anti- $\mathrm{H}$ in their circulation receive transfusions of blood that contains the $\mathrm{H}$ antigen (e.g., blood group $\mathrm{O}$ ), they are at risk of acute hemolytic transfusion reaction. Since their red blood cells (RBCs) do not react with anti- $\mathrm{A}$, anti- $\mathrm{B}$ and anti- $\mathrm{AB}$ antisera, they mimic $\mathrm{O}$ blood group in cell typing. The Oh individuals can only be transfused with autologous RBCs or blood from individuals of Bombay Oh phenotype which is very rare. We present our experience of detection of $\mathrm{H}$-deficient rare blood group Oh phenotype donors/patients in Iranian Blood Transfusion Organization (IBTO). The prevalence of Bombay blood group is rare in the Iranian general population. As this rare blood type is believed to be associated with incidence of consanguineous marriages, it is expected to be more prevalent in rural than in urban populations. We, thus expected the prevalence of Oh (Bombay) blood group to be high in consanguineous marriages in Iran.

\section{KARGER}

() 2018 S. Karger GmbH, Freiburg

Fax +497614520714 


\section{Material and Methods}

This is a descriptive study that was done in the Immunohematology Reference Laboratory of the IBTO, Tehran, Iran. The general population was covered and investigated through the Iranian National Rare Donor Program. Both the recipients and donor were included in the recent study over a period of 7 years (2009-2016). Donors were selected according to the blood banking norms. Initial $\mathrm{ABO}$ blood grouping was determined by tube method using commercially prepared antisera, anti- $\mathrm{A}$, anti- $\mathrm{B}$, anti- $\mathrm{AB}$ (Iranian Blood Research and Fractionation, Tehran, Iran). The presence of $\mathrm{RhD}$ antigen was determined by anti-D (Iranian Blood Research and Fractionation-IBRF and DIAGAST, Loos, France). Cell grouping and serum grouping were done using commercial blood grouping reagents. Although, antibody screening test is not part of routine serology donor testing in Iran but part of pre-transfusion testing, antibody screening test is performed on recipients' blood samples. All suspicious patients' blood samples showing 'blood group $\mathrm{O}$ with strong positive results (anti-H reacting at $37^{\circ} \mathrm{C}$ and AHG phase) in the antibody screening test, antibody identification panel (IBTO in-house 3-cell and 11-cell ID panel), and negative auto control results were tested for confirmation of Bombay blood group by tube method. In tube method, one drop of anti-H lectin and one drop of $5 \% \mathrm{RBC}$ suspension, washed in isotonic saline, solution were mixed, shaken to homogenize, and then centrifuged and checked for agglutination.

Adsorption/elution technique was used to confirm absence of A and B antigens and to rule out para-Bombay phenotype which carry weak antigens of A or B. RBCs of $\mathrm{H}$-deficient individuals were washed, reagent antibody added (antiA or anti-B), mixed, incubated at $4{ }^{\circ} \mathrm{C}$ for at least $2 \mathrm{~h}$, and packed. After washing, it was eluted at $-30^{\circ} \mathrm{C}$, and elute was tested with $\mathrm{A}$ or $\mathrm{B}$ cells.

Inhibition test was performed to check the secretory status of $45 \%$ of the subjects. These are subjects that we could reach for saliva collection, and their adsorption/elution test showed negative reactivity. Fresh saliva of these individuals was collected, boiled, centrifuged, and the supernatant was used for testing. In two tubes marked one and two, two drops each of anti-H lectin was added. Saliva and normal saline (each $100 \mu \mathrm{l}$ ) were added in each tube, mixed, and incubated. To this, $50 \mu \mathrm{l}$ of $5 \%$ cell suspension of known blood group O RBCs were added, mixed, incubated, and centrifuged. All the procedures were done according to AABB Technical Manual [2].

History of any consanguineous marriages among subjects' genetic parents was recorded in the index cases. All individual parents who volunteered to participate in the study were checked for the presence or absence of A, B, and $\mathrm{H}$ antigens

\section{Ethical Considerations}

This study was approved by the ethics committee of IBTO and health services. Individuals were asked to sign an informed consent form before blood samples were obtained. All terms of the Helsinki declaration were considered, and the personal information remained anonymous.

\section{Results}

Analysis of the results of over 7 million first-time blood donors and recipients in Iran during 2009-2016 showed that the most common ABO blood group was blood group O, with 2,520,000 (36\%) subjects. 56 (44.6\% female vs $55.4 \%$ male) Oh phenotypes (0.0008\%), including 42 (75\%) donor and 14 (25\%) patients were detected in the entire study group, consisting of blood donors and individuals identified through other incidental means. 50 (89.3\%) individuals were born out of consanguineous marriage.

19 (33.9\%) Oh phenotype individuals were identified during 2015 and 2016; this was the highest rate of detection within the study period. In 2013-2015, 15 (26.7\%), in 2011-2013, 18 (32.1\%) and in 2009 to 2010, 4 (7.1\%) of Oh individuals were detected.

Among blood group $\mathrm{O}$ donors detected on routine blood grouping from 2009 to 2016 , as many as $0.001 \%$ had Oh blood group. Among all 56 subjects in this study, 46 (82.1\%) were Rh+ versus $10 \mathrm{Rh}-(17.9 \%)$. When applying the adsorption/elution technique, there was no agglutination with A or B cells confirming these donors to be Bombay, and not para-Bombay phenotype. Saliva testing of $45 \%$ of the cases by inhibition assay showed agglutination and proved them to be non-secretor of $\mathrm{H}$ antigen (see table 1 for more serological test results).

The population distribution of rare $\mathrm{H}$-deficient blood group in Iran is shown in table 2. The Oh (Bombay) phenotypes were from 12 provinces. The maximum number of $\mathrm{Oh}$ individuals (10 donors and 4 patients) were identified in Tehran province that includes the metropolitan city of Tehran, capital of Iran, a city with close to 8.6 million inhabitants as well as adjacent small cities and neighboring rural areas which in total inhibits a population of above 14.5 million (table 2).

\section{Discussion}

The discovery of the rare 'Bombay phenotype' blood group, by Bhende et al. [3] was a vital event in the field of immunohematology. This phenotype was characterized by the absence of A, B, and $\mathrm{H}$ antigens on RBCs, and the serum of these persons had anti-A, anti- $\mathrm{B}$, and anti-H; thus these individuals had antibodies in plasma reacting with all $\mathrm{RBC} A B O$ phenotypes. These individuals were termed as homozygous recessive 'hh' or Bombay phenotype. They were typed as blood group $\mathrm{O}$ on normal $\mathrm{ABO}$ grouping but on cross-matching showed incompatibility to blood group $\mathrm{O}$. They were Lewis antigen-positive $(\operatorname{Le}(\mathrm{a}+))[3,4]$. In our recent study about $82 \%$ of the individuals were Le $(\mathrm{a}+)$.

More than 130 Bombay phenotypes have been reported in various parts of the world. Bombay phenotype is rare, since it occurs in about 1 in 10,000 individuals in India while it occurs in about $1 / 1,000,000$ individuals in Europe $[5,6]$.

In a study from South India, 13 Oh phenotypes $(0.048 \%)$ were detected of which 7 were males and 6 females. Among these $13 \mathrm{Oh}$ phenotypes, only 3 were $\mathrm{RhD}$-, and consanguinity among parents was observed in 10 cases (77\%) in a study amongst Bombay phenotypes [7].

The surprisingly high proportion of the Bombay phenotype caused by homozygosis is explained by the average inbreeding occurring even in well-mixed populations. This finding was supported by previous studies that the Bombay phenotype is more prevalent in populations with high rate of consanguineous marriage [8].

Balgir [9] concluded that the practice of endogamy and consanguinity were the main cause of the high prevalence of Bombay blood group. Consanguinity leads to increased homozygous expression of rare recessive genetic characters like the Bombay phenotype. A consanguinity among parents was observed in 50 cases $(89 \%)$ in the recent study. 
Table 1. Laboratory data of H-deficient individuals*

\begin{tabular}{lll}
\hline Laboratory results by saline-tube tests & & \\
\hline DAT & auto control test & Lewis phenotype \\
\hline Positive 1 (1.7\%) (IgG-/C3d+weak $)$ & positive 0 (0\%) & Le (a+b-) $46(82.2 \%)$ \\
Negative 55 (98.3\%) (IgG-/C3d-) & negative 56 (100\%) & Le (a-b-) 10 (17.8\%) \\
\hline
\end{tabular}

DAT $=$ direct antiglobulin test

*Number of suspicious cases considered for Oh confirmation: 64 . Number of suspicious cases that were not confirmed as Oh phenotype: 8.

Table 2. Population distribution of rare H-deficient individuals in Iran

\begin{tabular}{lllllll}
\hline Province & $\begin{array}{l}\text { Number of } \\
\text { donors (\%) }\end{array}$ & $\begin{array}{l}\text { Number of } \\
\text { patients (\%) }\end{array}$ & $\begin{array}{l}\text { Total number of } \\
\text { donors + patients }\end{array}$ & $\begin{array}{l}\text { Subjects from } \\
\text { urban population }\end{array}$ & $\begin{array}{l}\text { Subjects from } \\
\text { rural population }\end{array}$ & $\begin{array}{l}\text { Subjects from consan- } \\
\text { guineous marriages }\end{array}$ \\
\hline Tehran & $10\left(17.9 \%^{*}\right)$ & $4(7.1 \%)$ & $14(25 \%)$ & 9 & 5 & 10 \\
Fars & $7(12.5 \%)$ & $0(0 \%)$ & $7(12.5 \%)$ & 3 & 4 & 7 \\
Khorasan Razavi & $2(3.6 \%)$ & $1(1.8 \%)$ & $3(5.3 \%)$ & 1 & 2 & 3 \\
Semnan & $1(1.8 \%)$ & $0(0 \%)$ & $1(1.8 \%)$ & 0 & 1 & 1 \\
Bushehr & $3(5.4 \%)$ & $0(0 \%)$ & $3(5.4 \%)$ & 0 & 3 & 3 \\
Zanjan & $1(1.8 \%)$ & $0(0 \%)$ & $1(1.8 \%)$ & 0 & 1 & 1 \\
Sistan and Baluchestan & $1(1.8 \%)$ & $0(0 \%)$ & $1(1.8 \%)$ & 0 & 1 & 1 \\
Mazandaran & $5(8.9 \%)$ & $1(1.8 \%)$ & $6(10.7 \%)$ & 0 & 6 & 6 \\
East Azerbaijan & $3(5.4 \%)$ & $4(7.1 \%)$ & $7(12.5 \%)$ & 5 & 2 & 5 \\
Hormozgan & $1(1.8 \%)$ & $1(1.8 \%)$ & $2(3.6 \%)$ & 0 & 2 & 2 \\
Isfahan & $5(8.9 \%)$ & $2(3.6 \%)$ & $7(12.5 \%)$ & 1 & 6 & 7 \\
Yazd & $3(5.4 \%)$ & $1(1.8 \%)$ & $4(7.1 \%)$ & 1 & 3 & 4 \\
Total & $42(75 \%)$ & $14(25 \%)$ & $56(100 \%)$ & $20(36 \%)$ & $36(64 \%)$ & $50(89 \%)$ \\
\hline
\end{tabular}

* Percentages were rounded to one decimal place.

The Bombay phenotype is also reported from other countries in Asia like Japan [10], Malaysia [11], Sri Lanka [12], and Thailand [13]. In a small study, Ravanparvar et al. [14] found one Bombay blood type among three different population in Tehran. Furthermore Zanjani et.al. [15] reported the first FUT1 deletion in Iranian individuals. FUT2 deletion has been reported previously. The finding of two FUT1 novel alleles in Iranian people is indicative of mutation diversity in this gene.

Moreover, 7 individuals with Bombay phenotype in the US in an Indian family were reported in Yunis et al. [16]. In previous studies, cases of Bombay blood group in South Africa [11] and a large series of $\mathrm{H}$-deficient individuals (around 1:1,000) were reported in Reunion Island near Madagascar, indicating that Bombay blood group is mostly confined to South East Asian countries [17].

An acute hemolytic transfusion reaction will occur in patients with anti-H in their circulation if they receive transfusions of blood with the $\mathrm{H}$ antigen (e.g., blood group $\mathrm{O}$ ) [3].

Physicians should be aware of the management of such patients. In a report from Iran, a transfusion reaction occurred in a case of Bombay blood group patient because only forward grouping was performed in routine testing using a crude slide method and inappropriate documentation of cross-matching resulting in not detecting the Bombay group [18]. It is very important that a simple test like the antibody screening test be implemented as standard method of pre-transfusion testing, including both forward and reverse grouping so that no patient is missed or receives wrong blood, which could lead to serious hemolysis due to transfusion.

Implementing a complete standard pre-transfusion testing consisting of $\mathrm{ABO} / \mathrm{RhD}$ antibody screening test and avoiding reliance solely on a complete cross match test in the laboratory minimizes errors and makes certain that the right test is performed, the right results are obtained, and the right blood product is provided to the right patient at the right time.

It has been suggested that in developing countries where the antibody screening test is not part of a routine pre-transfusion test to reduce the risk of fatal hemolytic transfusion reaction, transfusion medicine departments or blood banks or blood donor centers should merge 'routine serum typing or reverse grouping confirmation' along with 'blood group $\mathrm{O}$ cell control in the reverse grouping procedure [19]. It is important to obtain accurate results in blood group serology tests of the donor and patient compatibility testing, and it is equally essential that the results are transcribed, collated, and interpreted correctly so that compatible blood products are issued. Errors in the blood bank laboratory can be due to technical failure in serological testing, insufficient procedures leading to misidentification of the samples, or misinterpretation of results [5].

In Iran, the immunohematology reference laboratory (IRL) of 
the IBTO attempts to confirm the blood group discrepancies and can also provide confirmation on blood donors with Bombay $\mathrm{Oh}$ phenotype as they maintained the national rare donor registry. It should be emphasized that due to the blood group rarity individuals with Bombay blood group (Oh) can either receive autologous blood or blood from an individual of Bombay phenotype only. If transfusion is required in such patients, IRL services are easily available nationwide with no cost option and with the potential advantage of a reduction in blood transfusion complication.

\section{Conclusion}

The present study shows the prevalence of $\mathrm{H}$-deficient blood groups in the Iranian general population to be $0.0008 \%$. Overall, the rare prevalence is possibly related to more homogenous and currently rare rate of consanguineous marriages in modern society of Iran. High prevalence is found wherever consanguineous mating is still highly followed in more traditional society, which remains a dominant risk factor. Even though feasibility and economics are still critical factors in a large population like Iran, in addition to donor education and awareness and the long distances the donors may have to travel, there is a need for establishing regional or main provincial rare donor registries. Advances in molecular research and technology in blood services may play a very important role in a developing country like Iran since it is still the matter of concern that individuals with rare blood groups like Bombay phenotype are missed and not truly identified and are at risk of being transfused with wrong blood group leading to hemolytic transfusion reactions and possible patient mortality. All blood banks in developing countries need to adopt up to date quality standards for performing basic blood grouping tests and stay away from antiquated methods in order to avoid life-threatening situations in recipients and donors of blood products. Programs for implementation of the standard pre-transfusion methods (voluntary antibody screening test) in hospital blood banking have been started through training on a national level in Iran, but it seems that they still need the due attention. The establishment of a Iranian hemovigilance system in 2010 has raised questions with respect to transfusion-related complications contributed to understanding the gaps in this area.

\section{Acknowledgments}

The authors would like to offer their thanks to technologists from IBTO's special serology laboratories and routine donor blood group laboratories for performing initial serology testing and referring the blood samples to IRL.

\section{Disclosure Statement}

The authors report no conflicts of interest.

\section{References}

1 Arend P: Early ovariectomy reveals the germline encoding of natural anti-A- and Tn-cross-reactive immunoglobulin $\mathrm{M}(\mathrm{IgM})$ arising from developmental O-GalNAc glycosylations. (Germline-encoded natural anti-A/Tn cross-reactive IgM). Cancer Med 2017;6: 1601-1613.

2 AAAB: Technical Manual, 15th ed. Bethesda, AABB, 2005. www.eqas.ir/pdf/lib/AABB\%20Technical\%20 Manual\%2015TH.pdf (last accessed October 18, 2018).

3 Bhende Y, Deshpande C, Bhatia H, Sanger R, Race R, Morgan W, Watkins W: A' new' blood group character related to the ABO system. Lancet 1952;1:903-904.

4 Mallick S, Kotasthane DS, Chowdhury PS, Sarkar S: Bombay blood group: is prevalence decreasing with urbanization and the decreasing rate of consanguineous marriage. Asian J Transfus Sci 2015;9:129-132.

5 Shrivastava M, Navaid S, Peethambarakshan A Agrawal K, Khan A: Detection of rare blood group, Bombay (Oh) phenotype patients and management by acute normovolemic hemodilution. Asian J Transfus Sci 2015;9:74-77.

6 Oriol R, Candelier JJ, Mollicone R: Molecular genetics of H. Vox Sang 2000;78(suppl 2):105-108.
7 Verma A, Vani K, Kumar IC, Bai DJ: Prevalence of Bombay blood group in a tertiary care hospital, Andhra Pradesh, India. Asian J Transfus Sci 2011;5: 57-58.

8 Wagner F, Flegel W: Polymorphism of the h allele and the population frequency of sporadic nonfunctional alleles. Transfusion 1997;37:284-290.

9 Balgir R: Detection of a rare blood group, 'Bombay (Oh) phenotype' among the Kutia Kondh primitive tribe of Orissa, India. Indian J Hum Genet 2007;13: 109-113.

10 Kaneko M, Nishihara S, Shinya N, Kudo T, Iwasaki H, Seno T, Okubo Y, Narimatsu H: Wide variety of point mutations in the $\mathrm{H}$ gene of Bombay and para-Bombay individuals that inactivate $\mathrm{H}$ enzyme. Blood 1997;90: 839-849.

11 Sathe M, Vasantha K, Mhaisalkar P, Gorakshakar A: Distribution of Bombay (Oh) phenotypes in India. J Indian Anthrop Soc 1988;23:277-280.

12 De Zoysa N: Bombay (Oh) phenotype among Sinhalese in Sri Lanka. Ceylon Med J 1985;30:29.

13 Sringarm S, Sombatpanich B, Chandanayingyong D: A case of Oh (Bombay) blood found in a ThaiðMuslim patient. Vox Sang 1977;33:364-368.
14 Ravanparvar N, Samiee S, Asadi G, Saheb JM, Lotfi P, Taherian D: The survey of prevalence of rare Bombay $(\mathrm{Oh})$ blood group among 3 different population in Tehran. Sci J Blood Transfus Organ 2004;1:43-49.

15 Zanjani D, Afzal Aghaee M, Badiei Z, Mehrasa R, Roodsarabi A, Khayyami M, Shahabi M: Molecular basis of Bombay phenotype in Mashhad, Iran: identification of a novel FUT1 deletion. Vox Sang 2016;111: 88-92.

16 Yunis EJ, Svardal JM, Bridges RA: Genetics of the Bombay phenotype. Blood 1969;33:124-132.

17 Le Pendu J, Gerard G, Vitrac D, Juszczak G, Liberge G Rouger P, Salmon C, Lambert F, Dalix A, Oriol R: Hdeficient blood groups of Reunion island. II. Differences between Indians (Bombay Phenotype) and whites (Reunion phenotype). Am J Hum Genet 1983; 35:484-496.

18 Shahshahani HJ, Vahidfar MR, Khodaie SA: Transfusion reaction in a case with the rare Bombay blood group. Asian J Transfus Sci 2013;7:86-87.

19 Dipta T, Hossain A: The Bombay blood group: are we out of risk? Mymensingh Med J 2011;20:536-540. 